\title{
Shape evolution and effect of organic additives in the electrosynthesis of $\mathrm{Cu}$ nanostructures
}

Francesca Pagnanelli

Department of Chemistry, Sapienza University of Rome, P.le Aldo Moro 5, 00185 Rome (Italy)

\section{Abstract}

Copper nanostructures were electrodeposited onto FTO in potentiostatic conditions at acidic $\mathrm{pH}$ without and with organic additives with different charge and structure: two cationic surfactants (hexadecyl trimethyl ammonium bromide and substituted deoxycholic acid), two anionic ones (sodium dodecyl benzene sulfonate and sodium dioctyl sulfosuccinate), and two neutral ones (saponin and deoxycholic acid). Shape evolution (assessed by image analyses of SEM micrographs) was determined for different copper concentrations $(0.001-0.1 \mathrm{M})$ and discharged charge $(Q=0.001-0.1 \mathrm{C})$ without additives. A common scheme was observed: sphere-like particles were observed firstly, then evolving towards branched structures, which undergo a gradual branch enlargement finally giving aggregate-like structures.

As for the effect of organics, negatively-charged additives promoted nucleation rather than growth of nanoparticles, while positively-charged additives enhanced growth of copper structures rather than new nucleation.

Keywords: electrosynthesis; Cu nanostructures; organic additives; acidic sulphate bath; FTO

*Corresponding Author: francesca.pagnanelli@uniroma1.it; Fax +3906490631 


\section{INTRODUCTION}

Recent enhancements in nanotechnology have evidenced new opportunities for $\mathrm{Cu}$ nanostructures in innovative applications [1].

Electrodeposition is generally used for the production of $\mathrm{Cu}$ nanostructure due to flexibility of conditions, simple operations, and low environmental impacts.

Electrodeposition is a multi-step process including: diffusion of metallic species from the bulk to the outer side of the double layer with eventual electrosorption, charge transfer with the formation of adatoms, diffusion of adatoms towards growing centres, and incorporation in deposited crystals [2].

The variety of morphologies also oriented towards the formulation of conceptual models that are alternative to direct atomic attachment. This is the case of aggregative growth mechanism, which assumes the formation of nano-clusters growing until self-limiting size. These nano-clusters then diffuse forming aggregates, further growing by direct atomic addition after re-crystallization [3].

According to the operating conditions, electrodeposition rate can be controlled by diffusion, or by kinetic of charge transfer, or both of them in mixed regimes: polyhedral compact crystallites are preferentially obtained in kinetic regimes, while diffusion limited aggregates with dendritic hierarchical structures were observed in diffusive regimes [4]. Dendrite formation is related to the creation of an ion depletion zone favouring further reduction on the protrusions. Pulsed electrodeposition [5] can be used for obtaining low sized nanoparticles with plasmonic resonance properties [6]. Dendritic nanostructures are indeed of interest for the development of new catalysts [7], sensors [8], and supercapacitors [9].

Additives can also modulate the characteristics of deposits by different mechanisms. In conventional electroplating applications, the use of additives is well established in order to promote levelling and brightening effects [10]. Both levellers and brighteners determine a 
reduction of grain size by different mechanisms. Sorption onto cathode can determine an increase of the overpotential (thus leading to a reduction of the critical nucleus size) [11] or can prevent surface diffusion of adatoms towards growing centres (thus reducing aggregation). In turn, complexation of metallic species in solution can affect both diffusion in the bulk (due to steric impediment) and charge transfer at the cathode interface (favouring or hindering new nucleation events).

No relation was identified until now between the chemical structure of additives and their effects in metal deposition and only empirical observations are available: as an example, typical levellers are recognised as polymeric cationic molecules with aromatic groups [12]. This lack of knowledge is mainly due to the different ways additives can interfere according to the controlling regime in electrodeposition: i.e. an additive affecting diffusion of electroactive species can maximise its effect under diffusive regime, while no significant effect should be expected if the charge transfer is limiting the overall kinetic. This can also lead to contrasting experimental findings and explanations.

Synthesis of copper nanostructures was mainly investigated in sulphate baths in acidic and basic conditions obtaining metallic [12] and oxide deposits [13, 14], respectively. According to the literature results, copper oxides nanoparticles were obtained for $\mathrm{pH}$ larger than 5 [13], but this limit can vary according to bath composition.

Focusing on the acid conditions, a wide spectrum of operating conditions was reported in the literature. Different substrates were used as working electrode such as copper [12], glassy carbon [15], gold [16], ITO [17], FTO [9] thus obtaining different nanoparticle morphologies [18]. Even bath composition was variable with some preferred mixtures (such as PEG with chloride ions, CTAB, and thiourea generating fine grained deposits) $[19,20]$ and many others less common showing levelling ability (such as picolic acid [21], benzyl-phenyl modified polyethyleneimine [22], ethoxyacetic alcohol and triethyl-benzyl- 
ammonium chloride [23], citric acid [24], ascorbic acid [25]), or not affecting deposit structure (such as sucrose [26], horse-chestnut extract [23], and saccharin [27]).

Some works tried to investigate additive mechanism of interaction by electrochemical characterization (such as cyclic voltammetry and impedance analysis) [28, 29], but without confirmation of the effects on the final morphological characteristics of obtained deposits.

As for copper electrodeposition in potentiostatic conditions onto FTO in acidic sulphate baths, different works have been published using high overpotential $[9,12,30]$.

No work was found performing electrodeposition at low overpotential onto FTO in acidic sulphate bath without or with additives.

In this work, potentiostatic deposition of $\mathrm{Cu}$ onto FTO at low overpotential in acidic conditions using sulphate bath was performed in different operating conditions addressing the effect of copper concentration, discharged charge, and addition of different organics on the morphology of the deposits.

This work presented different novelty aspects: this is the first experimental study in which copper structures were electrodeposited on FTO in potentiostatic conditions at low overpotential at acidic $\mathrm{pH}$ addressing the shape evolution of the deposits.

Another novel aspect is the investigation of the effect of six different additives in the same conditions performing morphological and electrochemical characterization in order to assess interaction mechanisms.

\section{MATERIALS AND METHODS}

Copper deposits were obtained by potentiostatic electrodeposition (-0.2 V versus $\left.E^{\circ} \mathrm{Ag} / \mathrm{AgCl}\right)$ by using aqueous solutions prepared by $\mathrm{CuSO}_{4}$ (analytical grade reagent from Carlo Erba) in $1 \mathrm{M} \mathrm{H}_{2} \mathrm{SO}_{4}$ (analytical grade reagent from Sigma Aldrich) without stirring.

The deposition process was performed at $25^{\circ} \mathrm{C}$ in three-electrode thermostatic cell with a Pt disk employed as counter electrode, an $\mathrm{Ag} / \mathrm{AgCl}$ saturated electrode used as reference electrode and Fluorine Tin doped Oxide (FTO) glass foils (Pilkinton NSG TEC A8, Xop 
Fisica, S.L.- Castello Spain) with an exposed area of $1 \mathrm{~cm}^{2}$ as working electrode. Before use, FTO cathodes were cleaned by immersion in acetone-water solution (1:1) in ultrasonic bath for $10 \mathrm{~min}$. The same spatial location of the three electrodes was maintained for all the performed tests. Working and counter electrode were placed vertically and in front to each other at a distance of $3 \mathrm{~cm}$. Amel 7060 potentiostat/galvanostat was used for the electrodeposition tests and other electrochemical characterization tests reported in Supplemental Material (cyclic voltammetries and open circuit voltage measurements).

Electrodeposition tests were arranged changing the copper concentration in solution and the duration of electrodeposition (measured as passing charge, $Q$ ) as reported in Table1.

Table 1 Operating conditions tested in potentiostatic electrodeposition of copper sulphate solution in $\mathrm{H}_{2} \mathrm{SO}_{4}$ medium without and with surfactant addition; [Cu]: initial copper concentration, Q: discharged charge; SAP: saponin; HDC: deoxycholic acid; SDBS: sodium dodecyl benzene sulfonate; AOT: sodium dioctyl sulfosuccinate; CTAB: hexadecyl trimethyl ammonium bromide; AdDC+: substituted deoxycholic acid

\begin{tabular}{|c|c|c|c|}
\hline$[\mathrm{Cu}](\mathrm{M})$ & $\mathrm{Q}(\mathrm{C})$ & Surfactant & $\begin{array}{c}\text { Surfactant } \\
\text { concentration }(\mathrm{M})\end{array}$ \\
\hline 0.001 & $0.001,0.006,0.01,0.02,0.06,0.1$ & - & - \\
\hline 0.01 & $0.001,0.006,0.01,0.02,0.06,0.1$ & - & - \\
\hline 0.1 & $0.001,0.006,0.01,0.02,0.06,0.1$ & - & - \\
\hline 0.01 & 0.1 & SAP & $0.0003,0.001$ \\
\hline 0.01 & 0.1 & HDC & 0.0001 \\
\hline 0.01 & 0.1 & SDBS & $0.0006,0.0015$ \\
\hline 0.01 & 0.1 & AOT & $0.0002,0.0056$ \\
\hline 0.01 & 0.1 & CTAB & $0.0003,0.010$ \\
\hline 0.01 & 0.1 & AdDC + & 0.001 \\
\hline
\end{tabular}


Experimental tests were performed without organic additives (first series) and with organic additives (second series). Organic additives were: hexadecyl trimethyl ammonium bromide (CTAB), sodium dodecyl benzene sulfonate (SDBS), sodium dioctyl sulfosuccinate (AOT), and saponin (SAP) (all analytical grade by Sigma Aldrich). All these surfactants were tested at two concentrations as detailed in Table 1, one below and one over the respective critical micellar concentration (CMC) according to the values reported in the literature: $0.0007 \mathrm{M}$ for saponin from Quillaja [31], $0.001 \mathrm{M}$ for SDBS [32], $0.0026 \mathrm{M}$ for AOT [33], and $0.001 \mathrm{M}$ for CTAB [34].

Two biliary acids were also tested: deoxycholic acid (HDC) (neutral in acid solution), and a substituted deoxycholic acid $\left(\mathrm{AdDC}^{+}\right)$, which is positively charged (structures in Table 2). After electrodeposition, working electrodes were washed with distilled water and ethanol in order to remove traces of salts and organics. The deposited nanoparticles were then analysed by Zeiss-Auriga SEM and the Matlab package for image analysis was used to determine size and density of copper nanoparticles. The size of copper particles is the equivalent diameter determined from the estimated 2D coverage area. 
Table 2 Organics additives used during potentiostatic electrodeposition of $\mathrm{Cu}$ on FTO: names (acronyms), charge, and chemical structure.

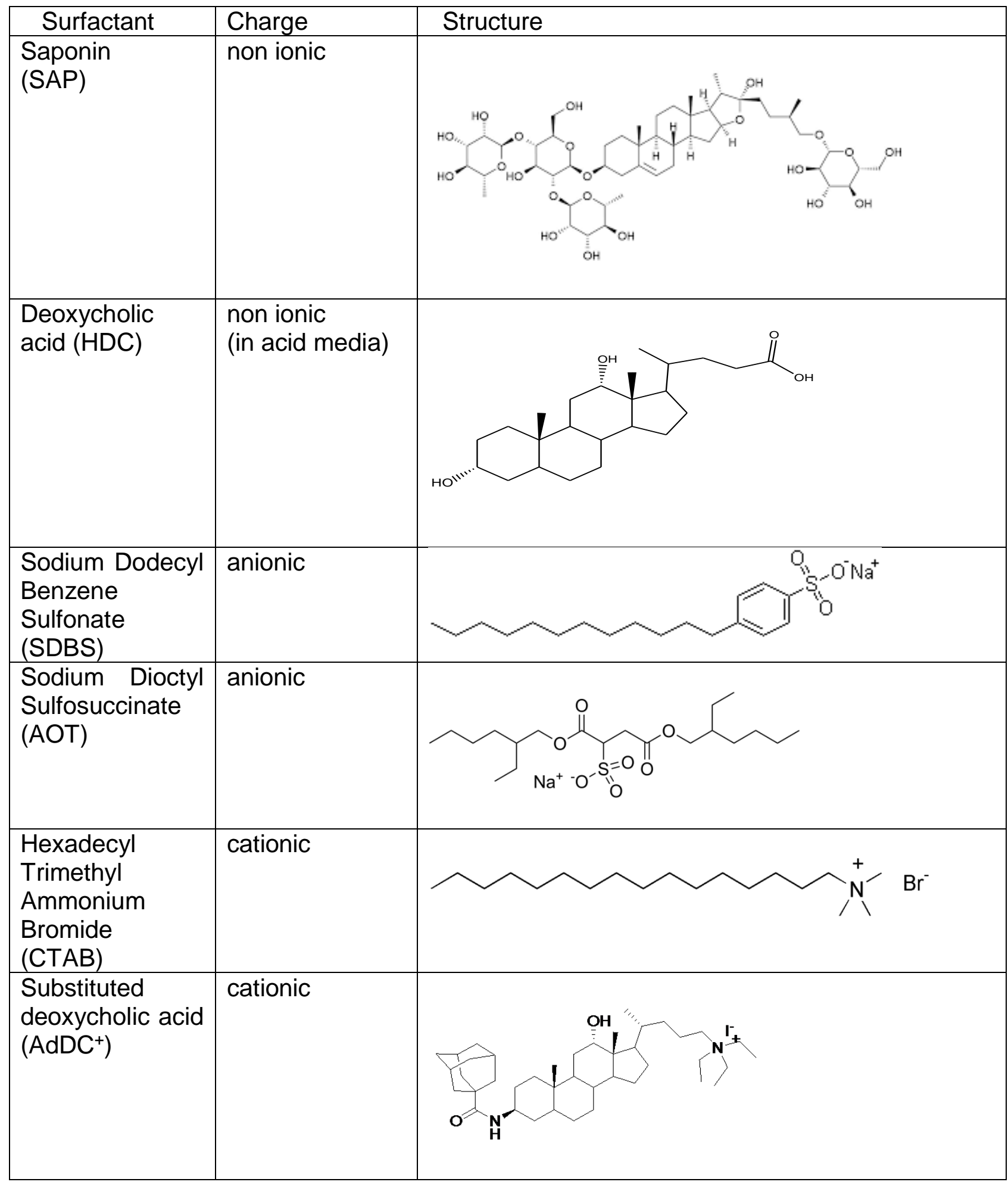




\section{RESULTS AND DISCUSSION}

\subsection{Electrodeposition of copper sulphate solutions}

Electrodeposition tests of copper sulphate solutions were performed in potentiostatic conditions at $-0.2 \mathrm{~V}$ vs $E^{\circ} \mathrm{Ag} / \mathrm{AgCl}$ for different initial copper concentrations $([\mathrm{Cu}]=0.001$, 0.010 and $0.100 \mathrm{M})$ and discharged charge $(Q=0.001-0.1 \mathrm{C})$.

Potential was chosen according to preliminary tests of cyclic voltammetry in a way that $\mathrm{Cu}$ was deposited and $\mathrm{H}_{2}$ formation was limited thus eliminating the effect of gas bubbles on deposit morphology [28].

Cyclic voltammetry for $0.01 \mathrm{M} \mathrm{Cu}$ concentration (Figure S1 in Supplemental Material) denoted that for negative potentials a cathodic current is generated due to $\mathrm{Cu}^{2+}$ reduction

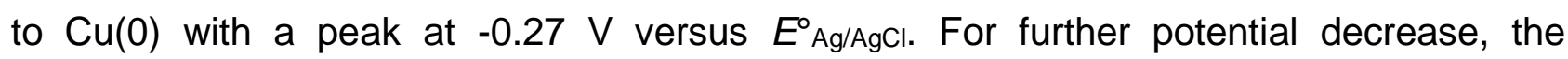
cathodic current also decreased due to diffusion control limiting the reduction of $\mathrm{Cu}^{2+}$. For potential more cathodic than $-0.5 \mathrm{~V}$ versus $E^{\circ} \mathrm{Ag} / \mathrm{AgCl}$, another increase of current can be observed due to hydrogen reduction.

Increasing the initial concentration of copper similar behaviours were observed with increased current peak (experimental data not reported here).

On the base of these results, potentiostatic deposition of copper was performed at $-0.2 \mathrm{~V}$ versus $E^{\circ} \mathrm{Ag} / \mathrm{AgCl}$ in order to have $\mathrm{Cu}^{2+}$ reduction and to limit $\mathrm{H}_{2}$ formation.

Performing electrodeposition in potentiostatic conditions a current transient occurred, which is generally characterised by an initial fast decay due to double layer charging, an increase of current due to the increase of the active area of new forming nanoparticles, and then a decrease of current due to the overlapping of hemispherical diffusion zones forming a planar layer. Deviations from this general behaviour have been conceptually explained by aggregative model considering an induction time due to nano-cluster formation (for long lasting initial decay), and low recrystallization rate limiting the growth by direct atom attachment (for the lack of maximum peak) [3]. 
The data of discharged charge versus elapsed time (Figure S2 in Supplemental Material) denoted significant differences in time scale for the tested conditions. In particular, increasing copper concentration a shorter time is required for discharging the same charge. Accordingly, the kinetic of discharge is faster increasing the copper concentration in solution.

Considering the variable dependence between charge and time, comparisons among the different operating conditions were made considering a scale of discharged charge instead of time.

For each copper concentration, the evolution of deposit morphology was quantitatively assessed by image analysis of SEM micrographs (Figures 1 and 2) evaluating both mean size and nanoparticle density for different discharged charge (Figures 3).

Initial characterization by EDX probe of SEM equipment denoted that the irregular black background observed in SEM images was made by FTO elements (Figure S3 in Supplemental Material), while the white nanoparticles (branched structures) were made by metallic copper (Figure S4 in Supplemental Material).

It should be noted that for statistical reasons (i.e. the need of having a sufficiently representative number of nanoparticles), image analysis was not performed only on SEM images reported in Figure 1, whose main aim is evidencing shape evolution. Image analysis of experimental data showed that at least 400 nanoparticles should be examined in order to have estimates of mean size and density, which are representative of the populations (elaboration not reported here). Accordingly, the number of SEM micrographs analysed for each condition can be variable depending on the density. Then some quantitative assessment reported in Figure 3 can apparently disagree with what observed in Figure 1. 
Figure 1 SEM images of Cu deposits obtained after the discharge of increasing values of charge ( $Q$ in vertical axis) for different initial copper concentrations ([Cu] in horizontal axis)

$[\mathrm{Cu}](\mathrm{M})$

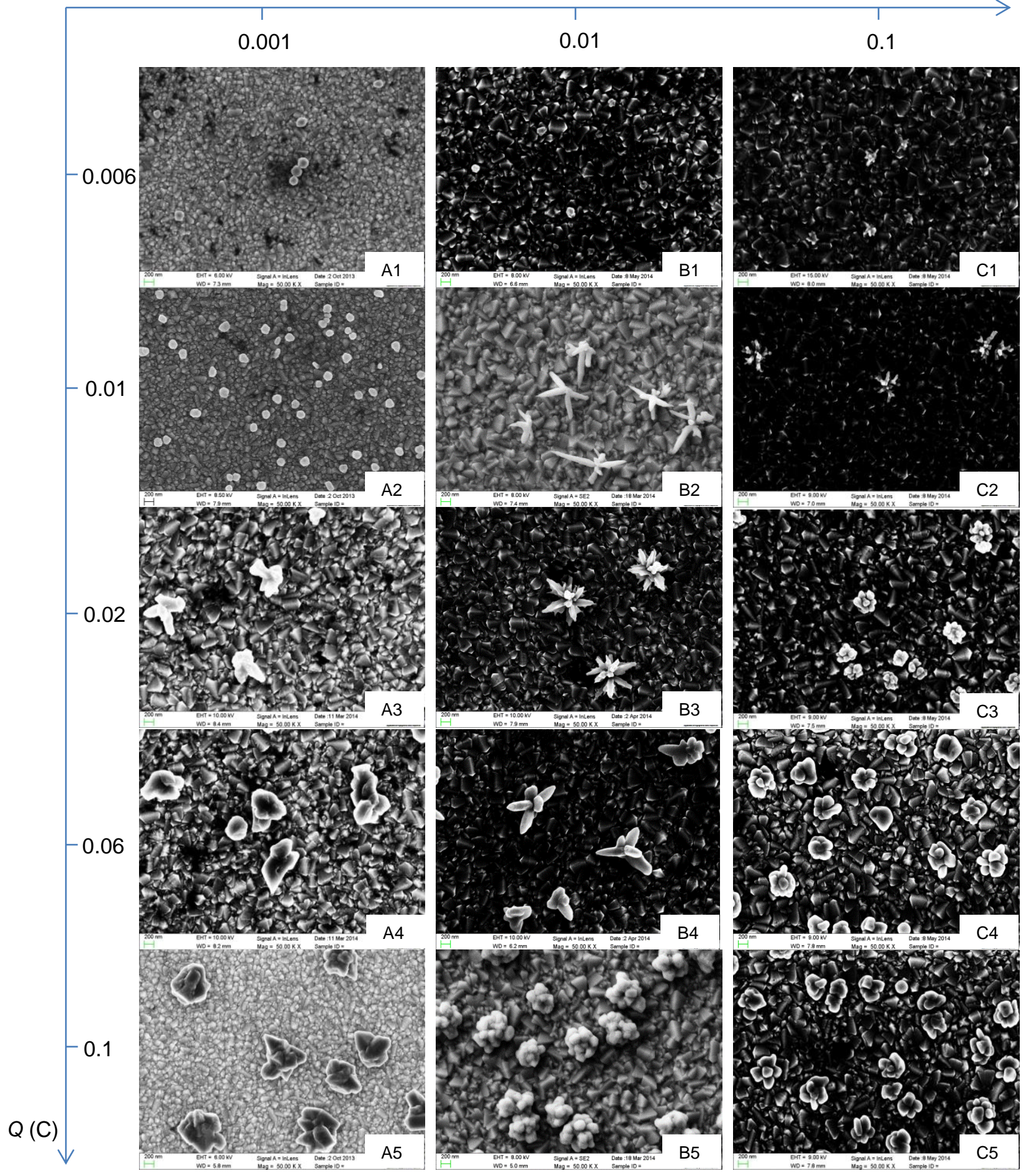


Fig 2 SEM images of Cu nanoparticles obtained after the discharge of $0.1 \mathrm{C}$ for different initial copper concentrations: $[\mathrm{Cu}]=0.001 \mathrm{M}(\mathrm{A}),[\mathrm{Cu}]=0.01 \mathrm{M}(\mathrm{B})$, and $[\mathrm{Cu}]=0.1$ $M(C)$

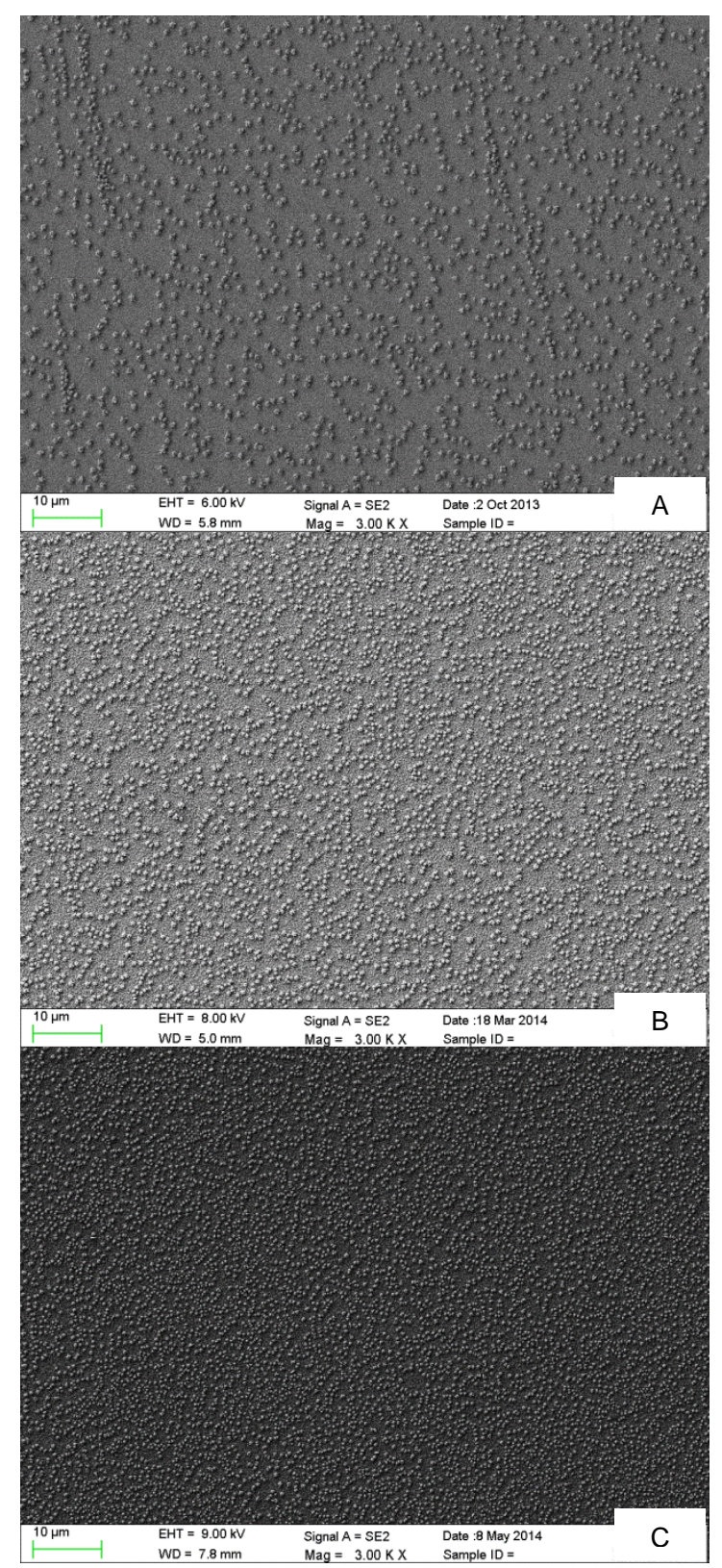


Figure 3 Mean size $(\mathrm{nm})$ and nanoparticle density (number of nanoparticle/ $/ \mathrm{m}^{2}$ ) estimated by image analysis of SEM micrographs recorded varying the initial copper concentrations $([\mathrm{Cu}])$ and the discharged charge $(Q)$
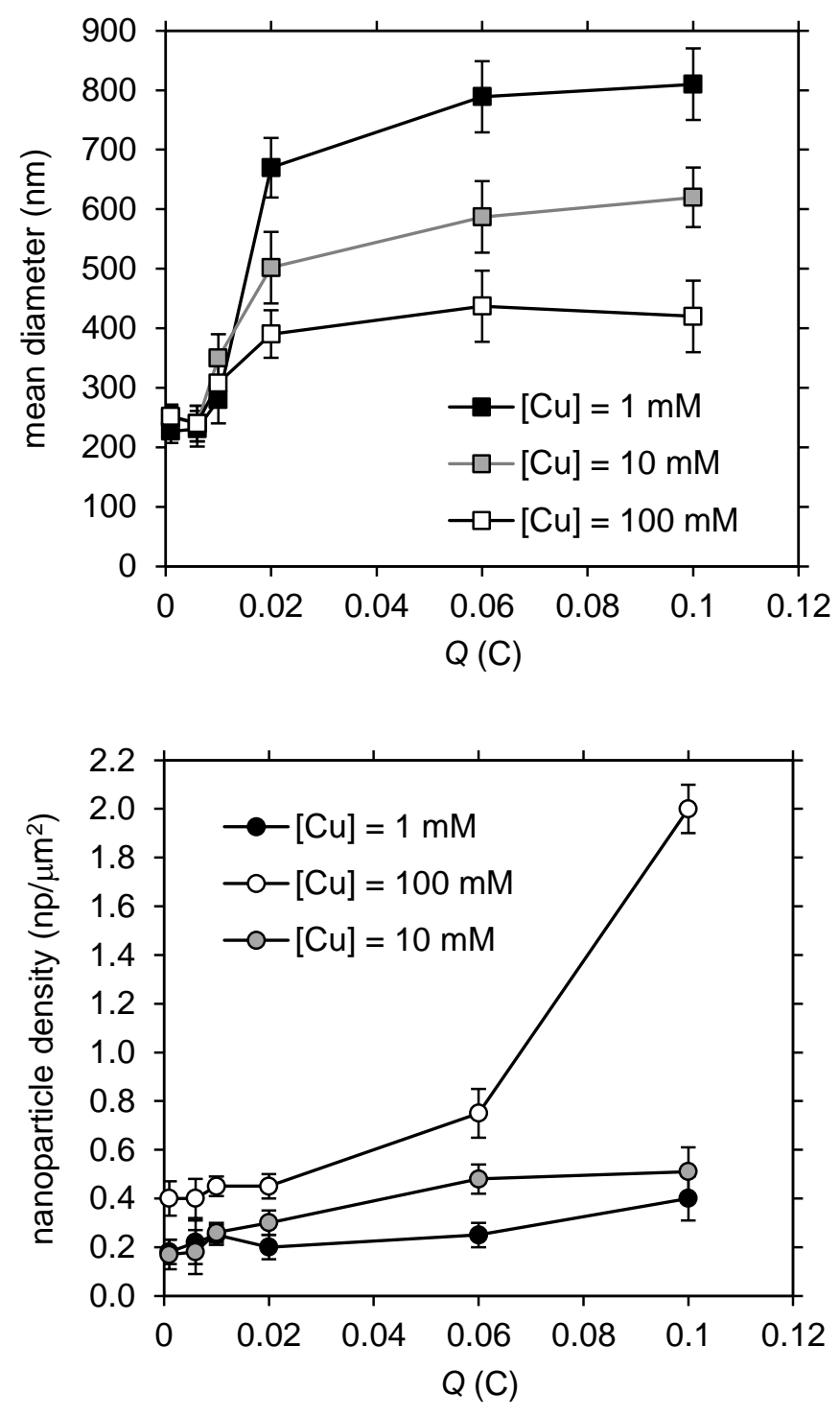

In Figure 1, deposit evolutions are reported as recorded by SEM images. It is possible to note that the same shape evolution can be observed for the different copper concentrations, but for different values of the discharge charge.

For $[\mathrm{Cu}]=0.001 \mathrm{M}$, in the first stages, sphere-like nanoparticles with mean size of $230 \pm$ $20 \mathrm{~nm}$ were observed (Figure $1 \mathrm{~A} 1$ ). Up to $Q=0.01 \mathrm{C}$ (Figure 1A2) an increase of 
nanoparticle density is recorded (from 0.18 to $0.25 \mathrm{np} / \mu \mathrm{m}^{2}$ ) without significant change in mean size (Figure 3). Then in the first phase new nucleation events occurred preferentially with respect to growth of already formed nanoparticles. Proceeding with electrodeposition, nanoparticles evolved towards irregular branched structures with increasing size (Figure 1A3). In this phase, growth of nanoparticles seems to be favoured with respect to new nucleation events. In fact, mean size increased while nanoparticle density remained constant (Figure 3). The same trend continued in the rest of electrodeposition characterized by a slight increase of mean size and relatively constant density. Considering the shape evolution, once branched structures formed, their growth continued with the gradual branch enlargement (Figure 1A4) finally giving aggregate-like structures (Figure 1A5).

Considering electrodeposition with $[\mathrm{Cu}]=0.01 \mathrm{M}$ (Figures 1B), a similar trend for increasing discharged charge was observed with three main differences.

The first difference is the recording of an intermediate structure between sphere-like nanoparticles and branched nanoparticles. In fact, after the formation of sphere-like nanoparticles (Figure 1B1) star-like deposits formed (Figure 1B2), which gradually evolved towards the previously observed branched structures (Figure 1B4) and, after branch thickening, resulted in aggregate-like structures (Figure 1B5). Then, except for the observation of these intermediate star-like structures, the same shape evolution was recorded: spheres, stars, branched nanoparticles, and finally aggregates.

The second difference concerns with the anticipation of such evolution with respect to the discharged charge scale. In fact, for $[\mathrm{Cu}]=0.001 \mathrm{M}$ spherical structures were observed at $Q=0.01 \mathrm{C}$, while increasing $\mathrm{Cu}$ concentration at $[\mathrm{Cu}]=0.01 \mathrm{M}$ star-like structures were already observed for the same $Q$.

The third difference is about the mean size and nanoparticle density: at $[\mathrm{Cu}]=0.01 \mathrm{M} \mathrm{a}$ reduced mean size ( $620 \pm 50 \mathrm{~nm}$ for $Q=0.1 \mathrm{C})$ and an increased nanoparticle density ( 0.51 
$\pm 0.01 \mathrm{np} / \mu \mathrm{m}^{2}$ ) was obtained with respect to the lower investigated concentration (800 \pm $60 \mathrm{~nm}$ and $0.40 \pm 0.09 \mathrm{np} / \mu \mathrm{m}^{2}$, respectively for $Q=0.1$ C) (Figures 1-3).

For the highest investigated level of copper concentration $[\mathrm{Cu}]=0.1 \mathrm{M}$ (Figures $1 \mathrm{C}$ ), starlike structures were already present at the first stages (Figure 1C1). These structures evolved according to the same mechanism described above by thickening of the star branches, finally giving aggregates. The evolution of shape resulted then further anticipated in the $Q$ scale, confirming a relation between concentration and rate of appearance of the different characteristic stages. As for the trend in mean size and density, a further reduction of mean size $(420 \pm 60 \mathrm{~nm})$ and increase in nanoparticle density $\left(2.0 \pm 0.1 \mathrm{np} / \mu \mathrm{m}^{2}\right)$ were evidenced for this concentration as reported in Figure 3.

The experimental findings regarding the effect of concentration on nanoparticle density can be explained considering the effect of increasing overpotential, which in this case means increasing the metal concentration in solution working at constant potential: in particular, the overpotential changes due to $\mathrm{Cu}$ concentration estimated by Nernst equation are $0.03 \mathrm{~V}$ (from 0.001 to $0.01 \mathrm{M}$ ) and $0.05 \mathrm{~V}$ (from 0.01 to $0.1 \mathrm{M}$ ).

According to the thermodynamic view of Milchev [11] increasing the overpotential determined a reduction of the size of the critical nucleus: then increasing the overpotential more nanoparticles formed but with decreased size for the same discharged charge. The same effect can be also explained in kinetic terms considering that the nucleation rate is related to the overpotential [5].

As for the shape evolution, after nucleation, nanoparticles start to grow consuming copper ions in the limiting layer. If nucleation rate is sufficiently fast (high overpotentials), growth is controlled only by diffusion of metal ions from bulk solution to the boundary layer. In such conditions, diffusion layer aggregates typically form with structures deviating from regular shapes observed in kinetic regimes [22]. Different works reported in the literature evidenced the formation of dendritic Cu leaves of large size (around $1 \mu \mathrm{m}$ ) using high 
overpotential as typical diffusion layer aggregates $[9,12,30]$. Such dendritic structures are not observed in the present work probably due to the low overpotential determining mixed regimes.

High concentration of sulphate ions in solution $(1 \mathrm{M})$ can also contribute in lowering $\mathrm{Cu}$ diffusion from bulk to cathode. In fact, sulphate can form complexes in solution with copper ions, thus limiting their diffusion to the cathode. Star-like branched structures were already observed [14] with addition of electrolyte salts $\left(\mathrm{K}_{2} \mathrm{SO}_{4}, \mathrm{Na}_{2} \mathrm{SO}_{4}\right.$ or $\left.\left(\mathrm{NH}_{4}\right)_{2} \mathrm{SO}_{4}\right)$ at $0.1 \mathrm{M}$ concentration during the $\mathrm{Cu}$ electrodeposition onto ITO electrodes in sulphate bath and potentiostatic conditions (-0.6 V vs $\left.E^{\circ} \mathrm{Ag} / \mathrm{AgCl}\right)$. On the other side, without adding these salts, aggregates of polyhedral nanoparticles were obtained as in the final stage of electrodeposition. The Authors explained these findings by the lower diffusion of sulphate complexes of $\mathrm{Cu}$ with respect to aquo-complexes. Then sulphate complexes of copper in 1 $\mathrm{M} \mathrm{H}_{2} \mathrm{SO}_{4}$ solution used in the present work could be responsible for limited diffusion of copper in solution and then for the formation of branched structures.

Previous discussion is based on classical model describing electrodeposition according to direct atomic attachment. Nevertheless, the final shape of the deposits as aggregate-like structures also recalls the mechanism of aggregative growth. In particular, looking at the first steps of nanoparticle formation in conditions of low kinetics (then low $\mathrm{Cu}$ concentration) the sphere-like nanoparticles seem to be aggregates of more units (see Figures $1 \mathrm{~A} 2$ and $1 \mathrm{~B} 1)$. After formation, these aggregates started to grow by direct attachment giving star-like structures, which continued to grow by direct atomic attachment giving an enlargement of star branches and the formation of aggregate-like structures in the last phase.

Chronoamperometric curves related to electrodeposition tests evidenced a monotonous decrease of current during time also deviating from Cottrell equation (see for examples the curves reported in Figure S5 of Supplemental Material). The lack of the maximum peak 
denoted the occurrence of phenomena that are different from those commonly expected. An interpretation of such trend can be found in the aggregative growth model, which relates decaying current transients to low re-crystallization rate limiting the growth by direct attachment with no relevant increase of active surface area [3].

Then both shape evolution and current transients could be in favour of aggregative growth mechanism operating in the investigated conditions.

\subsection{Electrodeposition of copper sulphate solutions in presence of additives}

Copper electrodeposition was performed adding different surfactants at concentration larger and lower the respective Critical Micellar Concentration, CMC. Different organics were chosen in order to assess the effect of charge and chemical structures. In particular, two cationic surfactants (hexadecyl trimethyl ammonium bromide, CTAB, and substituted deoxycholic acid, AdDC+), two anionic ones (sodium dodecyl benzene sulfonate, SDBS, sodium dioctyl sulfosuccinate, AOT), and two neutral ones in acidic conditions (saponin, SAP, and deoxycholic acid, HDC) were used. As reported in Table 2, chosen additives

presenting the same charge are characterized by very different chemical structures: according to the availability for each class of charge, one long chain and one branched additive were chosen.

In Figures 4 and 5, SEM images were reported for the case of surfactant addition at concentration larger than $\mathrm{CMC}$, when micelles of surfactant monomers are expected to form (except for the two biliar acids that were used only at one concentration), while in Figure 6 SEM images for concentration lower than CMC were reported.

Quantitative analysis (as mean size and particle density) is reported in Figure 7. 
Fig 4 SEM micrographs of Cu deposits $(Q=0.1 \mathrm{C})$ with the addition of deoxycholic acid (HDC), saponin (SAP), sodium dodecyl benzene sulfonate (SDBS), sodium dioctyl sulfosuccinate (AOT), hexadecyl trimethyl ammonium bromide (CTAB), and substituted deoxycholic acid ( $\left.\mathrm{AdDC}^{+}\right)$at concentration larger than $\mathrm{CMC}$

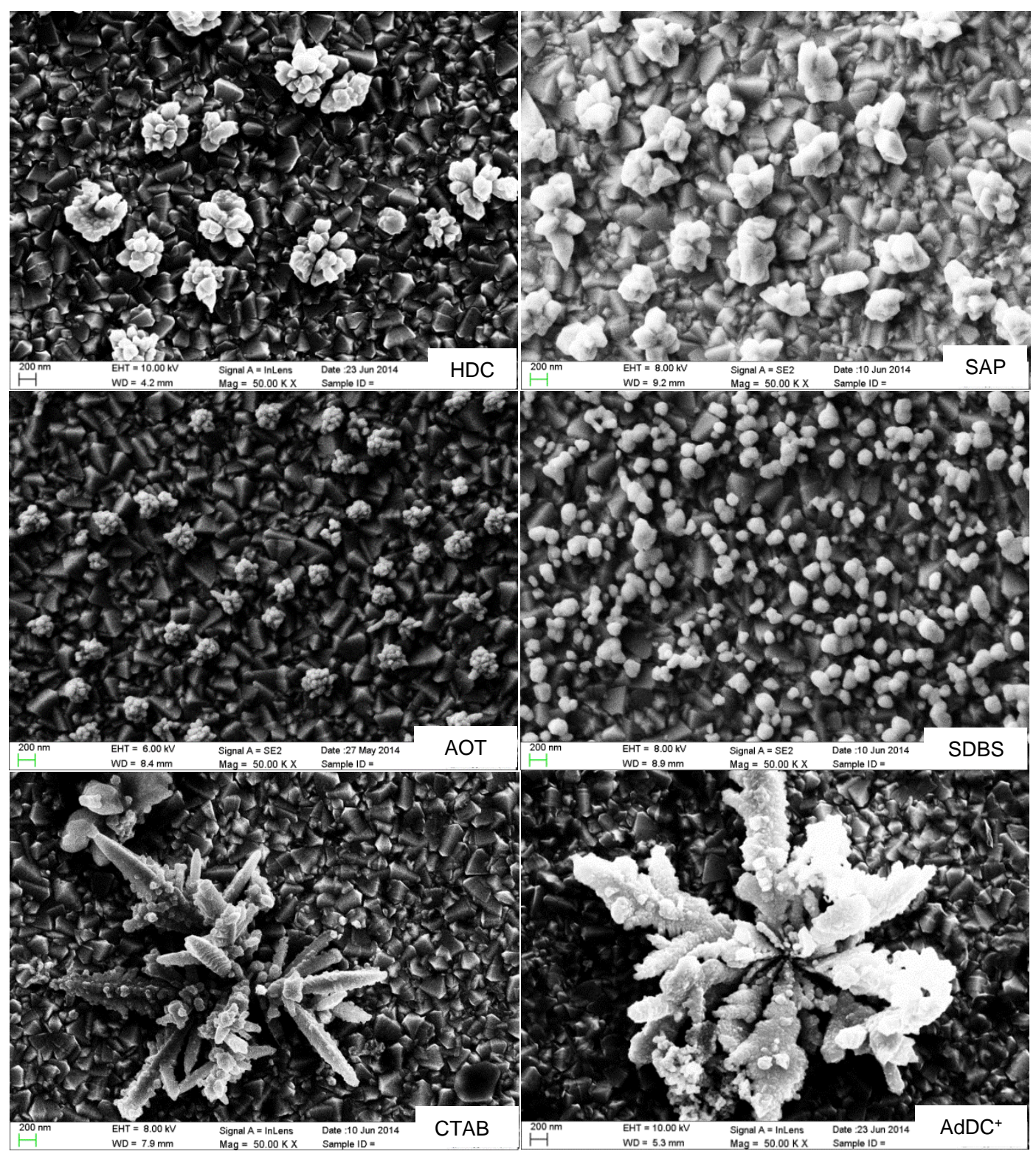


Fig 5 SEM micrographs of Cu deposits $(Q=0.1 \mathrm{C})$ with the addition of deoxycholic acid (HDC), saponin (SAP), sodium dodecyl benzene sulfonate (SDBS), sodium dioctyl sulfosuccinate (AOT), hexadecyl trimethyl ammonium bromide (CTAB), and substituted deoxycholic acid ( $\left.\mathrm{AdDC}^{+}\right)$at concentration larger than $\mathrm{CMC}$

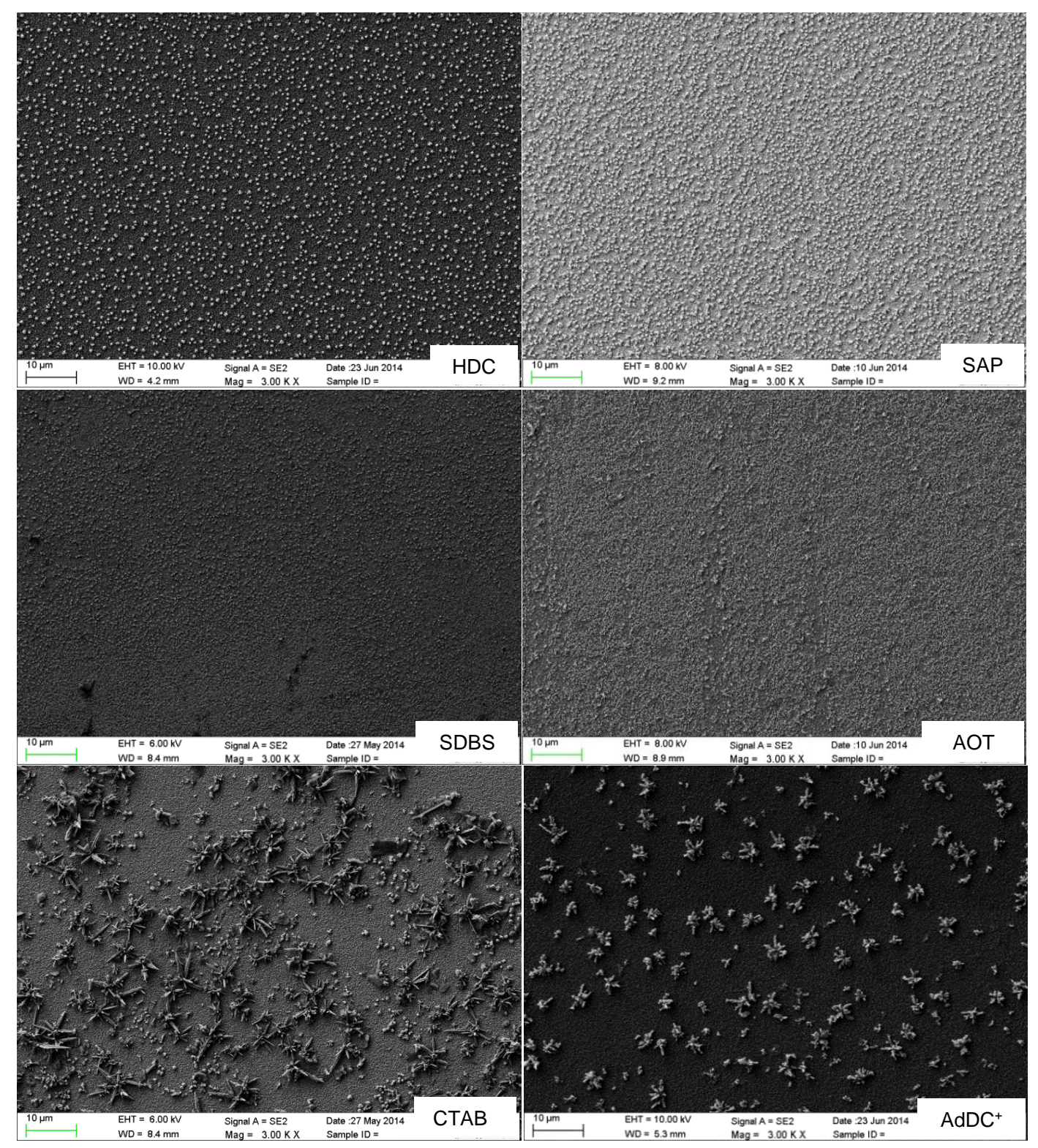


Fig 6 SEM images of $\mathrm{Cu}$ deposits $(Q=0.1 \mathrm{C})$ with the addition of hexadecyl trimethyl ammonium bromide (CTAB), saponin (SAP), sodium dioctyl sulfosuccinate (AOT), and sodium dodecyl benzene sulfonate (SDBS) at concentration lower than CMC

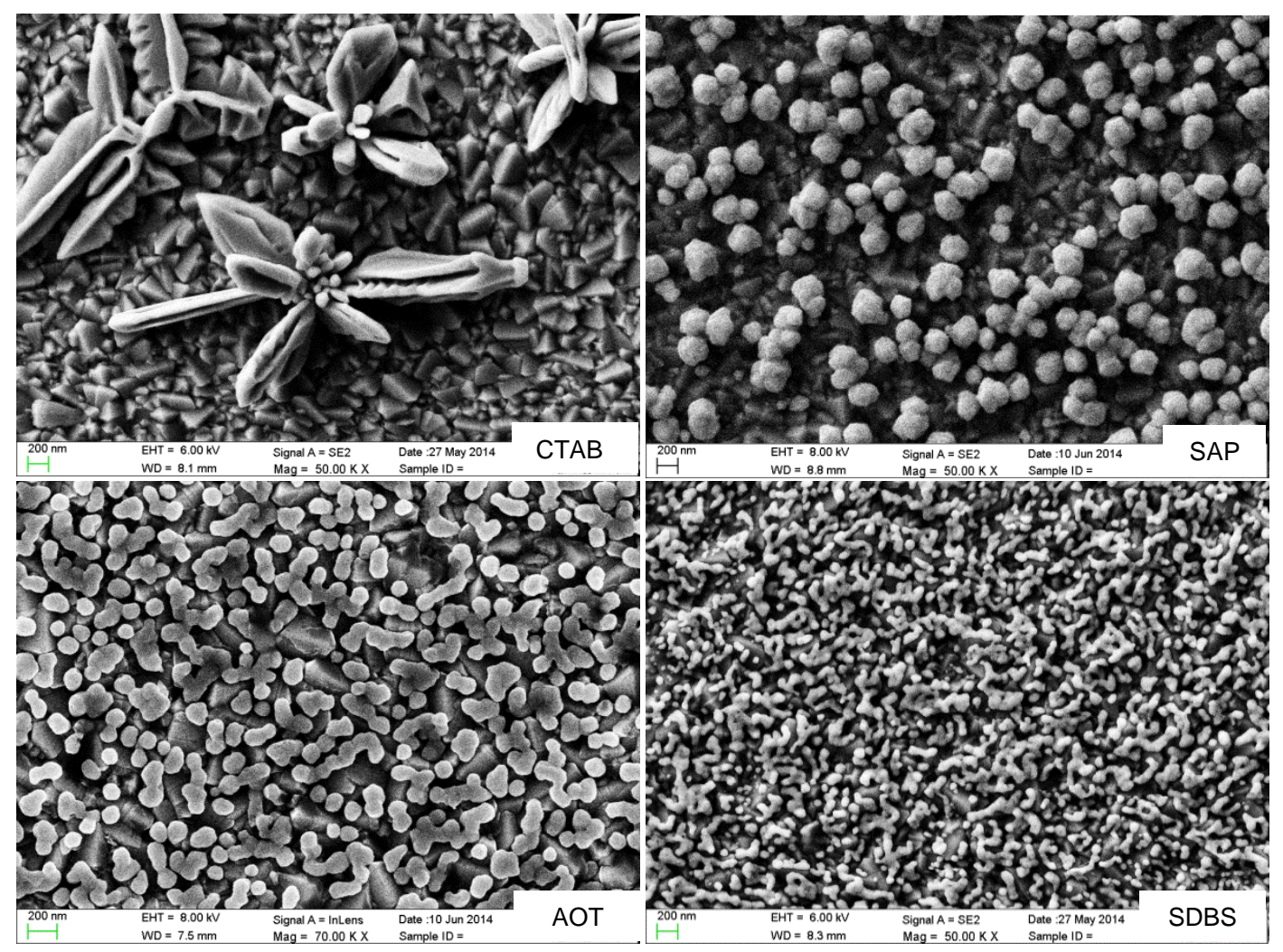

Experimental findings showed that when neutral additives (SAP and HDC) were added no significant difference was observed about shape, size and density of copper deposits (Figures 4, 5 and 7).

On the contrary, when anionic surfactants (SDBS and AOT) were added to the system, a dramatic change of deposit morphology was found (Figures 4, 5 and 7). In both cases, a significant decrease in mean size was observed associated to an increase of density. As for the shape, low-sized aggregated structures were observed in the case of AOT, while spherical nanoparticles were present adding SDBS. 
Fig 7 Mean size $(\mathrm{nm})$ and nanoparticle density (number of nanoparticle/ $\mu \mathrm{m}^{2}$ ) estimated by image analysis of SEM micrographs recorded for $[\mathrm{Cu}]=0.01 \mathrm{M}$ and $Q=0.1 \mathrm{C}$ without surfactant addition (TQ) and with the addition of different additives (sodium dioctyl sulfosuccinate (AOT), sodium dodecyl benzene sulfonate (SDBS), deoxycholic acid (HDC)) at concentration larger and lower than the critical micellar concentration ( $>\mathrm{CMC}$ and $\angle \mathrm{CMC}$, respectively)
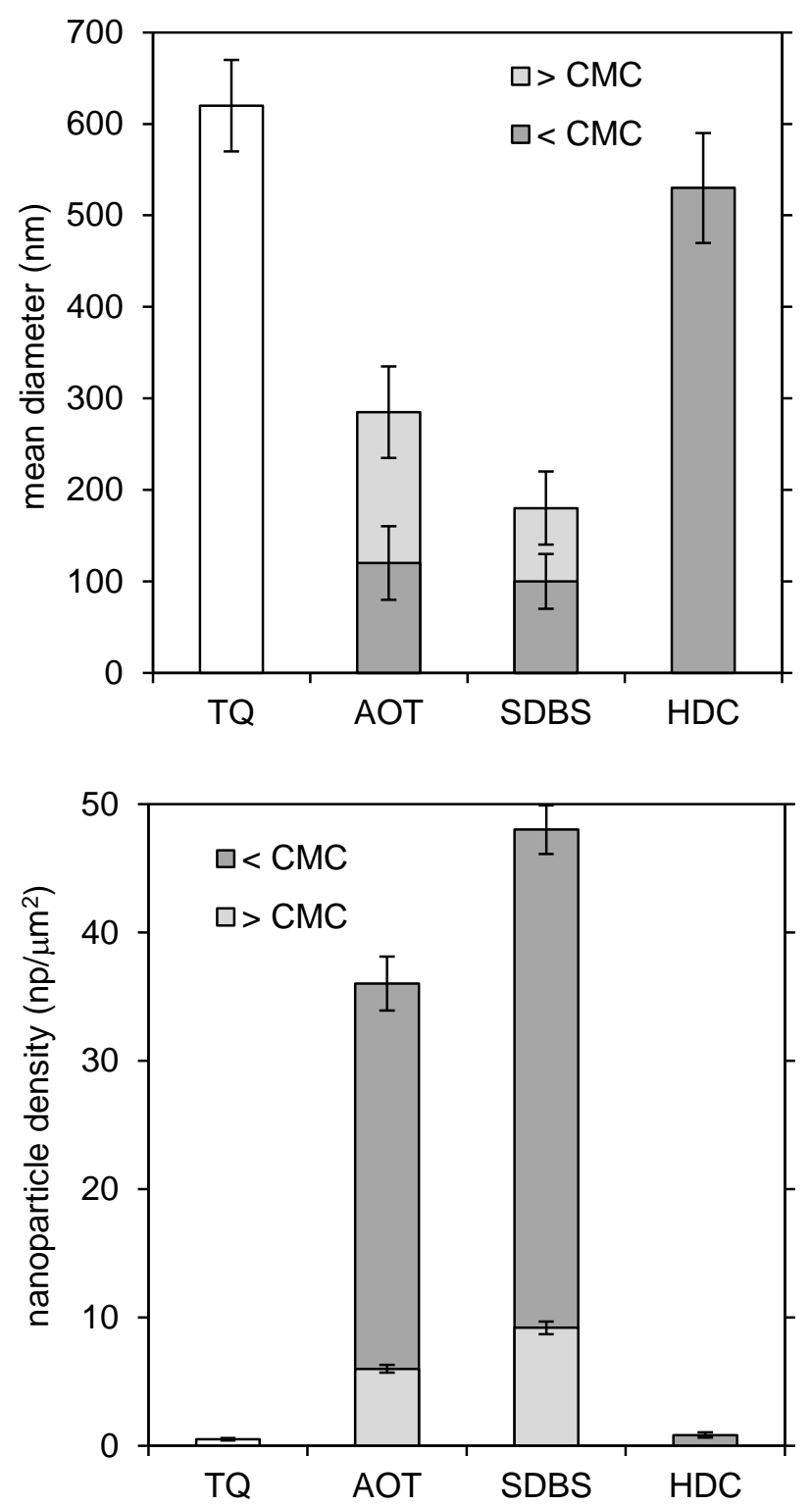
These experimental findings evidenced that both anionic surfactants interact with the system promoting nucleation rather than growth of nanoparticles.

This behaviour can be explained according to different mechanisms:

- Additive-electrode interactions limiting adatom (or cluster) diffusion towards growing centres and coalescence

- Specific interactions between surfactants and deposited nanoparticles: these interactions blocked further growth of nanoparticles thus favouring new nucleation events. Considering that electrodeposited nanoparticles are protruding points with increased potential they can be preferential sites for additive sorption.

- Interactions between surfactant and metallic species in solution giving complexes more susceptible to reduction thus enhancing nucleation rate.

As for cationic surfactants $(\mathrm{CTAB}$ and $\mathrm{AdDC}+)$ their addition determined a significant change in the morphology of deposits (Figure 4). Spherical and aggregate-like structures leave the place to flower-like structures with large size $(>1000 \mathrm{~nm})$ and low density. Adding cationic surfactants then opposite effects than adding anionic surfactants are observed.

This finding can be explained according to different mechanisms:

- Interactions with ions in solution limiting their diffusion by complexation or simply by screening of the electrode

- Interactions with the electrodic surface depressing new nucleation events: the few points where electrocrystallization occurred, continued growing under diffusioncontrolled conditions (giving diffusion controlled branched aggregates).

Few literature works can be used as references to discuss these data due to the great variability of experimental conditions used, not allowing direct comparison.

In the electrodeposition of $\mathrm{CuCl}_{2}$ solution in acidic conditions $\left(\mathrm{H}_{2} \mathrm{SO}_{4}\right)$ onto copper electrode [35] various additives (cetyltrimethylammonium bromide, CTAB, sodium dodecyl 
sulphate, SDS, or polyvinylpyrrolidone, PVP) were used denoting an additive-depending morphology with flowers (CTAB), dendrites (SDS), and sphere-like (PVP) hierarchical morphologies. No information about possible mechanism of differentiation due to the addition of the various additives was furnished.

$\mathrm{Cu}$ deposition onto $\mathrm{Cu}$ substrate in sulfuric bath with addition of chloride and CTAB [12] showed that for low overpotential CTAB inhibited $\mathrm{Cu}$ nucleation by sorption onto the cathode. The simultaneous presence of chloride ions is responsible for the formation of cupric chloride complexes adsorbed onto the cathode enhancing $\mathrm{Cu}$ reduction. Unfortunately, no experiment was performed without chloride in the system.

In order to assess the effect of additive concentration, tests were performed also at concentration lower than CMC (Figure 6) denoting similar effects.

Nevertheless, considering mean size and density of nanoparticles an amplification of the effect of anionic surfactants was observed having lower mean size and higher density than in the case in which these same surfactants are added at concentration larger than CMC. Then aiming at drastically reducing mean size of deposits (and consequently increasing density) anionic surfactant should be used at concentration lower than CMC.

As for saponin the reduction of surfactant concentration in solution determined a reduction of mean size and an increase of density. The observed effect could be due to the same mechanism described for anionic surfactant. Nevertheless, a clear explanation of this finding is not possible at this stage due to the complex structure of saponin, the uncertain value of $\mathrm{CMC}$ and the complete lack of literature work regarding this additive.

Tests of electrochemical characterization were performed in order to investigate the mechanism of interaction of additives.

Cyclic voltammetry tests were performed in the same conditions of electrodeposition using positive and negative additives that denoted the main effect on the morphology of the deposits. 
The presence of negative additives (SDBS and AOT) (Figure S6 in Supplemental Material) shifted the peak of $\mathrm{Cu}^{2+}$ reduction towards more negative potentials (about $0.2 \mathrm{~V}$ more negative values) denoting an inhibition of the reduction process. This increased potential could be due to interactions between additives and electrodes or additives and $\mathrm{Cu}^{2+}$ ions in solution. In order to distinguish between these two options, open circuit voltage (OCV) of the solutions prepared with increasing additive concentrations was measured: for both negative additives a decrease of OCV of about $0.2 \mathrm{~V}$ was registered evidencing an interaction between additive and electrodes (Figure S7 in Supplemental Material).

According to these tests, it can be argued that negative surfactants, despite their charge, interact with the electrode giving an extra resistance requiring increased potential for copper discharge.

These observations are in agreement with the observed effect of negative additives on deposit morphology (i.e. reduction of grain size) because the interaction between electrode and additive inhibits surface diffusion of adatoms (or clusters) towards growing centres and their coalescence.

The presence of positive additives (CTAB and $\left.A d D C^{+}\right)$did not evidenced significant differences in reduction peaks in cyclic voltammetry (Figure S6 in Supplemental Material) nor substantial interaction with electrodes in OCV measurements (Figure S7 in Supplemental Material).

According to these additional characterizations it can be deduced that the main effect of positive additives (giving high dimension flower-like aggregates) is as screening agents in solution hindering $\mathrm{Cu}^{2+}$ ion diffusion and favouring the growth of protrusions.

\section{CONCLUSIONS}

Electrosynthesis of metallic nanostructures is attracting interest due to the simplicity of operations and versatility of products. Nevertheless, the complexity of phenomena simultaneously occurring during electrocrystallization is not yet formalized to an extent 
allowing the tuning of deposit characteristics by selection of operating conditions. Then, research and development activities are still in the phase of data collection in order to evidence trends and inspire new conceptual models.

Experimental findings reported in this work evidenced the complexity of the system even in simple reference conditions without additives, without other anions except sulphates, and with low overpotential giving not relevant $\mathrm{H}_{2}$ formation. When organic additives were added relevant changes were observed mainly depending on the charge of the additives and not on their structures. Considering the multistep nature of electrodeposition phenomenon and the variety of regimes occurring, the relation between additive charge and deposit morphology found in the present work is far away from being a general rule, having validity in the investigated conditions, in which mixed regimes probably occurred. Nevertheless, this is like arranging another piece in the puzzle of electrosynthesis formalization for straightforward tailoring of deposits.

\section{AKNOWLEDGEMENT}

The Author thanks Prof. Luciano Galantini of the Department of Chemistry of Sapienza University for the biliar acids kindly furnished and used for the tests.

\section{REFERENCES}

[1] Ustarroz J, Hubin A, Terryn H (2016) Handbook of nanoparticles. Springer International Publishing, Switzerland.

[2] Budevski E, Staikov G, Lorenz WJ (1996) Electrochemical phase formation and growth Willey-VHC, Weinheim.

[3] Ustarroz J, Hammons JA, Altantzis T, Hubin A, Bals S, Terryn H (2013) A generalized electrochemical aggregative growth mechanism. J Am Chem Soc 135:11550-11561. 
[4] Altimari P, Pagnanelli F (2016) Electrochemical nucleation and three-dimensional growth of metal nanoparticles under mixed kinetic-diffusion control: model development and validation. Electrochim Acta 206:119-126.

[5] Pagnanelli F, Schiavi PG, Bellagamba M, Moscardini E, Granata G, Toro L (2015) Pulsed electrodeposition of cobalt nanoparticles on copper substrate: quantitative assessment of the effects of the operating parameters. Electrochim Acta 155: 228-235.

[6] Doménech-Carbó A, Galian RE, Aguilera-Sigalat J, Perez-Prieto J (2016) Handbook of Nanoparticles. Springer International Publishing, Switzerland

[7] Taleb A, Xue Y, Dubot $P$ (2013) Self organized gold nanoparticles as new nanoelectrocatalyst templates for surface nanostructuring. J Electroanal Chem 693:60-66. [8] Guadagnini L, Ballarin B, Tonelli D (2013) Dendritic silver nanostructures obtained via one-step electrosynthesis: effect of nonanesulfonic acid and polyvinylpyrrolidone as additives on the analytical performance for hydrogen peroxide sensing. J Nanopart Res 15:1971.

[9] Jeyasubramanian K, Gokul Raja TS, Purushothaman S, Kumar MV, Sushmitha I (2017) Supercapacitive performances of $\mathrm{MnO} 2$ nanostructures grown on hierarchical $\mathrm{Cu}$ nano leaves via electrodeposition. Electrochim Acta 227:401-409.

[10] Oniciu L, Muresan L (1991) Some fundamental aspects of levelling and brightening in metal electrodeposition. J Appl Electrochem 21:565-574.

[11] Milchev A (2002) Electrocrystallization: fundamentals of nucleation and growth. Kluwer Academic Publishers, Dordrecht.

[12] Bozzini B, D'Urzo L, Re M, De Riccardis F (2008) Electrodeposition of Cu from acidic sulphate solutions containing cetyltrimethylammonium bromide (CTAB). J Appl Electrochem 38:1561-1569.

[13] Wan L, Wang Z, Yang Z, Luo W, Li Z, Zou Z (2010) Modulation of dendrite growth of cuprous oxide by electrodeposition. J Crystal Growth 312:3085-3090. 
[14] Mignani A, Ballarin B, Boanini E, Cassani MC (2014) Simple one step electrochemical preparation of copper nanostructures. Electrochim Acta 115:537-545.

[15] Grujicic D, Pesic B (2002) Electrodeposition of copper: the nucleation mechanisms. Electrochim Acta 47:2901-2912.

[16] Radisic A, Ross FM, Searson PC (2006) In situ study of the growth kinetics of individual island electrodeposition of copper. J Phys Chem B 110:7862-7868.

[17] Wei C, Wu G, Yang S, Liu Q (2016) Electrochemical deposition of layered copper thin films based on the diffusion limited aggregation. Scientific Reports 6 Article number: 34779 .

[18] Yang CJ, Lu FH (2013) Shape and size control of Cu nanoparticles by tailoring the surface morphologies of TiN-coated electrodes for biosensing applications. Langmuir 29:16025-16033.

[19] Bonou L, Eyraud M (2002) Influence of additives on Cu electrodeposition mechanisms in acid solution: direct current study supported by non-electrochemical measurements. Electrochim Acta 47:4139-4148.

[20] Pasquale MA, Gassa LM, Arvia AJ (2008) Copper electrodeposition from an acidic plating bath containing accelerating and inhibiting organic additives. Electrochim Acta 53:5891-5904.

[21] Portela AL, Teijelo ML, Lacconi GI (2006) Mechanism of copper electrodeposition in the presence of picolinic acid. Electrochim Acta 51:3261-3268.

[22] Bozzini B, D'Urzo L, Mele C (2007) A novel polymeric leveller for the electrodeposition of copper from acidic sulphate bath: a spectroelectrochemical investigation. Electrochim Acta 52:4767-4777.

[23] Muresan L, Varvara S (2000) The effect of some organic additives upon copper electrowinning from sulphate electrolytes. Hydrometallurgy 54:161-169. 
[24] Mandke MV, Pathan HM (2012) Electrochemical growth of copper nanoparticles: Structural and optical properties. J Electroanal Chem 686:19-24.

[25] Abouzeid FM (2015) Influence of vitamin C on copper electrorefining from acidic solution of copper sulfate. Asian J Chem 27:4337-4343.

[26] Raja M, Subha J, Ali FB, Ryu SH (2008) Synthesis of copper nanoparticles by electroreduction process. Materials and Manufacturing Processes 23:782-785.

[27] Quinet M, Lallemand F, Ricq L, Hihu JY, Delobelle P, Arnould C, Mekhalif Z (2009) Influence of organic additives on the initial stages of copper electrodeposition on polycrystalline platinum. J Electrochim Acta 54:1529-1536.

[28] Burke LD, O'Connell AM, Sharna R, Buckley CA (2006) Involvement of a metastable surface state in the electrocatalytic, electrodeposition and bath additive behaviour of copper in acid solution. J Appl Electrochem 36:919-929.

[29] Vaduva CC, Vaszilcsin N, Kellenberger A (2011) Effect of aromatic amines on the diffusion layer thickness during the copper electrodeposition from acid bath. Chemical Bulletin of "Politehnica" University of Timisoara 56(70):75-80.

[30] Nikolic ND, Popov KI, Pavlovic LJ, Pavlovic MG (2007) Determination of critical conditions for the formation of electrodeposited copper structures suitable for electrodes in electrochemical devices. Sensors 7:1-15.

[31] Dias Ribeiro B, Sales Alviano D, Weingart Barreto D, Zarur Coelho MA (2013) Functional properties of saponins from sisal (Agave sisalana) and juá (Ziziphus joazeiro): critical micellar concentration, antioxidant and antimicrobial activities. Colloid Surface A 436:736-743.

[32] Zhang L, Zhao C, Jiang Y, Wang Y, Yang W, Cheng T, Zhou G (2018) Effect of sodium dodecyl benzene sulfonate on morphology and structure of calcium silicate hydrate prepared via precipitation method. Colloid Surface A 540:249-255. 
[33] Ankitkumar IF, Eleftheria A, Paschalis A, Tsianou M (2015) Self-assembly of sodium bis(2-ethylhexyl) sulfosuccinate in aqueous solutions: modulation of micelle structure and interactions by cyclodextrins investigated by small-angle neutron scattering $\mathrm{J}$ Mol Liq 210:125-135.

[34] Zdziennicka A, Szymczyk K, Krawczyk J, Janczuk B (2012) Critical micelle concentration of some surfactants and thermodynamic parameters of their micellization. Fluid Phase Equilibr 322-323:126-134.

[35] Dong H, Wang Y, Tao F, Wang L (2012) Electrochemical fabrication of shapecontrolled copper hierarchical structures assisted by surfactants. J Nanomat Article ID 901842. 